\title{
Mortalidad perioperatoria de pacientes sometidos a apendicectomías en el régimen contributivo de Colombia
}

\author{
Perioperative mortality of patients undergoing appendectomies in \\ the contributory regime in Colombia
}

\author{
Jimmy Santiago Castellanos-Méndez $\mathbb{D}_{+}$Nicolle Simmonds-Campbell $^{2} \mathbb{D}$, \\ Giancarlo Buitrago-Gutiérrez ${ }^{3}$ D
}

1 Estudiante de medicina, Semillero de Investigación Clínica, Facultad de Medicina. Instituto de Investigaciones Clínicas. Departamento de Cirugía. Universidad Nacional de Colombia, Bogotá D.C., Colombia.

2 Estudiante de medicina, Semillero de Investigación Clínica, Facultad de Medicina. Instituto de Investigaciones Clínicas. Departamento de Cirugía. Universidad Nacional de Colombia, Bogotá D.C., Colombia.

3 Médico, MSc, PhD. Instituto de Investigaciones Clínicas. Departamento de Cirugía. Universidad Nacional de Colombia, Bogotá D.C., Colombia.

\section{Resumen}

Introducción. La mortalidad perioperatoria se considera una de las herramientas clave para medir la fuerza del sistema de salud de un país en el área quirúrgica. Actualmente, no se cuenta con las cifras totales de las apendicectomías realizadas en nuestro pais, a pesar de ser un procedimiento quirúrgico extremadamente común.

Métodos. Estudio de cohorte retrospectivo con datos de pacientes de todas las edades, reportados como afiliados al sistema de salud contributivo de Colombia y a quienes se les realizó una apendicectomía entre el $1^{\circ}$ de enero de 2011 y el 31 de diciembre de 2016. La información se obtuvo de bases de datos nacionales y se procesó a partir del cálculo de variables, como la tasa de mortalidad perioperatoria y el volumen quirúrgico, estimando una tasa de mortalidad a treinta días, por sexo, grupo etario y región geográfica.

Resultados. Entre las 231.570 apendicectomías que se identificaron, se halló una mayor tasa de mortalidad perioperatoria en el grupo etario de hombres mayores de 80 años con 17,3 \%. La región oriental presentó la menor tasa de mortalidad con 0,01 \%. El mayor volumen quirúrgico se presentó en el año 2012 y el menor en el año 2016.

Discusión. La tasa de mortalidad de apendicectomía en diferentes grupos etarios es similar a la de otros países de ingresos medios y bajos, en tanto que la mayor heterogeneidad de la tasa de mortalidad entre las diferentes regiones, se percibe en los grupos de menores de un año y de mayores de 65 años. Este estudio se constituye como una aproximación inicial a la tasa de mortalidad en apendicectomías realizadas en Colombia.

Palabras clave: apendicectomía; laparoscopia; grupos de edad; mortalidad; Colombia.

Fecha de recibido: 21/05/2020 - Fecha de aceptación: 27/05/2020

Correspondencia: Nicolle Simmonds-Campbell. Diagonal 182 No 20 - 17 Apto 619, Bogotá, D.C., Colombia. Teléfono: 3188080087 Correo electrónico: nsimmondsc@unal.edu.co

Citar como: Castellanos-Méndez JS, Simmonds-Campbell N, Buitrago-Gutiérrez G. Mortalidad perioperatoria de pacientes sometidos a apendicectomías en el régimen contributivo de Colombia. Rev Colomb Cir. 2021;36:91-7. https://doi.org/10.30944/20117582.668

Este es un artículo de acceso abierto bajo una Licencia Creative Commons - BY-NC-ND https://creativecommons.org/licenses/by-ncnd/4.0/deed.es 


\begin{abstract}
Introduction. Perioperative mortality is considered one of the key tools to measure the strength of a country's health system, in the surgical area. Currently, there are no total figures for appendectomies performed in our country, despite being an extremely common surgical procedure.

Methods. Retrospective cohort study with data from patients of all ages, reported as affiliated to the contributory health system in Colombia and who underwent an appendectomy between 01/01/2011 and 12/31/2016. The information, which was obtained from national databases, was processed by calculating variables such as perioperative mortality rate and surgical volume, estimating a 30-day mortality rate, by sex, age group and geographic region.
\end{abstract}

Results. Among the 231,570 appendectomies that were identified, a higher perioperative mortality rate was found in the age group of men older than 80 years with $17.31 \%$. The eastern region had the lowest mortality rate with $0.01 \%$. The highest surgical volume occurred in 2012 and the lowest in 2016.

Discussion. The mortality rate from appendectomies in different age groups is similar to that of other low- and middle-income countries, while the greater heterogeneity of the mortality rate between the different regions is seen in the groups of children under one year of age and older over 65 years old. This study constitutes an initial approximation to the mortality rate in appendectomies performed in Colombia.

Keywords: appendectomy; laparoscopy; age groups; mortality; Colombia.

\section{Introducción}

El dolor abdominal es la causa más común de consulta en los servicios de urgencia y ocasiona la mayoría de las emergencias abdominales, generalmente debidas a apendicitis ${ }^{1-3}$. Esta patología tiene un riesgo estimado de presentación del $8 \%$ y su tratamiento quirúrgico, la apendicectomía, corresponde al procedimiento más frecuentemente realizado por los cirujanos generales ${ }^{2}$. Tiene una mayor ocurrencia en hombres y es poco común en los extremos de la vida, aunque con mayor mortalidad (hasta del $8 \%$ ) en la población de edad avanzada ${ }^{6-8}$.

Desde el año 2000, la incidencia de apendicitis en países recientemente industrializados de Latinoamérica ha superado a la de muchos países desarrollados, registrando más de 150 casos por 100.000 personas por año. Por esto, las instituciones rectoras de la salud deben preparar su infraestructura de atención médica, para lograr el correcto diagnóstico y manejo de los pacientes con apendicitis y así lograr una disminución de la morbimortalidad asociada ${ }^{9}$.
La Comisión de Cirugía Global de Lancet define la mortalidad perioperatoria como uno de los indicadores centrales, para medir la fuerza del sistema de salud en el área quirúrgica de un país; aun así, muchos países de ingresos medios y bajos no cuentan con estudios poblacionales para la adecuada medición de este indicador ${ }^{10}$. En Colombia, no contamos con estudios suficientes que evalúen cuáles son las cirugías más frecuentemente realizadas en la población general ni sus características asociadas.

El objetivo del presente estudio fue determinar la tasa de mortalidad perioperatoria en Colombia, en pacientes a quienes se les practicó apendicectomía durante el periodo comprendido entre los años 2011 y 2016, así como determinar el volumen quirúrgico del periodo 2012 - 2016 y describir sus características, con el propósito de incentivar el desarrollo de futuros estudios y políticas, que permitan mejorar la calidad en la atención de los pacientes que son sometidos a dicho procedimiento. 


\section{Métodos}

\section{Tipo de estudio y población}

Se realizó un estudio retrospectivo de cohortes de individuos de todas las edades, que fueron sometidos a algún tipo de apendicectomía, y que se encontraban afiliados a alguna de las entidades promotoras de salud (EPS) del régimen contributivo, entre el año 2011 y 2016. Se incluyeron solamente las EPS que enviaron y pasaron la revisión de calidad de información para el estudio de la suficiencia de la Unidad de Pago por Capitación (UPC), realizado por el Ministerio de Salud y Protección Social. Se excluyeron a los individuos que salieron del régimen contributivo en los 30 días posteriores a la realización del procedimiento.

Se utilizó la base de datos para el estudio de la suficiencia de la UPC desde el 1 de enero del 2011 al 31 de diciembre del 2016 para 4 tipos de procedimientos en apendicectomías, definidos por la Clasificación Única de Procedimientos en Salud (CUPS), como apendicectomía (471100), apendicectomía por laparoscopia (471110), apendicectomía por perforación con drenaje de absceso, liberación de plastrón y/o drenaje de peritonitis localizada (471200) y apendicectomía con drenaje de peritonitis generalizada (471300).

También se consultó la base de datos de estadísticas vitales del Departamento Administrativo Nacional de Estadística (DANE), para la identificación de las personas fallecidas en el periodo de estudio. Por último, se utilizó el Sistema Integral de la Protección Social (SISPRO) y el Sistema de Gestión de Datos (SG9), mediante la conexión Cubo RIPS, para obtener el número de personas afiliadas anualmente a cada una de las EPS que cumplían con las características ya descritas. Se tomaron únicamente los datos del mes de julio de cada año y, teniendo en cuenta que estos datos solo se encuentran disponibles desde el año 2012, se analizaron únicamente desde dicho año hasta el 2016. Para todo lo anterior, las bases de datos fueron anonimizadas.

Este es un estudio sin riesgos según la Resolución 8430 de 1993 del Ministerio de Salud de Colombia, el cual además fue aprobado por el
Comité de ética de la Facultad de Medicina de la Universidad Nacional de Colombia.

\section{Variables}

La tasa de mortalidad perioperatoria se definió como el número de muertes que ocurrieron el día de la cirugía o dentro de los primeros 30 días después de la cirugía, dividido entre el número de admisiones para el mismo procedimiento. Se estimó la tasa general y la tasa específica por edad, sexo y región, para lo cual las regiones geográficas se definieron según la división dispuesta por el DANE, como son: Región Atlántica, que incluye los departamentos de Atlántico, Bolívar, Cesar, La Guajira, Magdalena, Córdoba y Sucre; Región Central, conformada por los departamentos de Antioquia, Caldas, Caquetá, Huila, Quindío, Risaralda y Tolima; Región Pacífica, conformada por los departamentos de Valle del Cauca, Cauca, Chocó y Nariño; Región Oriental, conformada por los departamentos de Norte de Santander, Boyacá, Cundinamarca, Meta y Santander; Otros departamentos, conformada por Arauca, Casanare, Putumayo, San Andrés, Amazonas, Guainía, Guaviare, Vaupés y Vichada; y Región Bogotá, D.C.

De otra parte, se definieron 8 grupos etarios, así: grupo 1, menores de 1 año; grupo 2 , de 1 a 5 años; grupo 3, de 5 a 10 años; grupo 4, de 10 a 18 años; grupo 5, de 18 a 49 años; grupo 6, de 50 a 64 años; grupo 7 , de 65 a 80 años, y grupo 8, mayores de 80 años.

\section{Análisis}

Con la información obtenida de las bases de datos, se organizó y procesó con Stata $14^{\circledR}$, calculando inicialmente el total de apendicectomías realizadas, la edad, sexo y región geográfica de los pacientes. Se incluyeron además los datos del registro de fallecimientos del DANE, lo que permitió calcular la tasa de mortalidad perioperatoria por grupos en regiones y grupos etarios, para posteriormente ser estimada. Así mismo, se calcularon los procedimientos realizados entre el 2012 y el 2016 con la base de datos de suficiencia de la UPC, para posteriormente definir el número de afiliados por las EPS, incluidas en el estudio, a través de la utilización de la base de datos SISPRO incluyendo 
el cálculo del volumen quirúrgico, dividiendo el número de procedimientos realizados por cada 1000 personas afiliadas a estas EPS.

\section{Resultados}

Nuestro estudio identificó 231.570 apendicectomías realizadas entre el 2011 y el 2016, de las cuales el tipo de cirugía más comúnmente llevada a cabo fue la apendicectomía abierta (no asociadas a peritonitis), con un total de 158.303 procedimientos (68,3\%). La apendicectomía por laparoscopia fue la realizada menos frecuentemente con 14.179 casos $(6,1 \%)$. En la población pediátrica, clasificada entre las edades de 0 a 18 años, se realizaron 65.317 procedimientos $(28,2 \%)$, y la gran mayoría, es decir 166.253 (71,8 \%) se realizaron en población adulta. Se encontró una leve tendencia hacia el sexo masculino, con 116.945 casos (51\%). La región en la que se practicaron más apendicectomías fue Bogotá, D.C., con 78.748 cirugías (34\%), y, en segundo lugar, la región central con $53.182(23 \%)$ procedimientos.

En la tasa de mortalidad perioperatoria por sexo, estimada por cada grupo etario, se hizo evidente una diferencia significativa en los mayores de 80 años, encontrándose una tasa del 17,3\% en hombres frente a una tasa del $12,5 \%$ en mujeres. Asimismo, se encontró otra brecha en el grupo etario correspondiente a los menores de un año, siendo mayor en mujeres, con una tasa de 3,4 \%, en comparación con los pacientes del sexo masculino con un 2,9\% (tabla 1).

En los grupos etarios (tabla 2), encontramos una elevada tasa de mortalidad perioperatoria en los mayores de 80 años (14,4\%), seguido por el grupo comprendido entre los 65 a 80 años (5,5\%) y en menores de 1 año $(3,1 \%)$. Los pacientes entre 10 y 18 años tuvieron la menor tasa de mortalidad $(0,02 \%)$.

No se halló registro de muertes perioperatorias para apendicectomías en los departamentos de Chocó, Amazonas, Guainía, Guaviare y Vaupés, departamentos que, a excepción del Chocó, corresponden a la división por región de "otros departamentos", por lo tanto, se excluyen de los resultados finales. La región atlántica, presentó una tasa de mortalidad mayor en los grupos etarios de 1 a 5 años (0,4 \%), de 10 a 18 años (0,04\%), de 50 a 64 años (1,5 \%) y de 65 a 80 años (9,1\%), sin embargo, tuvo la menor tasa de mortalidad perioperatoria en menores de un año $(2,0 \%)$ en comparación con Bogotá, D.C., donde se presentó la mayor tasa de mortalidad $(6,8 \%)$.

Para las personas de 18 a 50 años y mayores de 80 años, la tasa de mortalidad perioperatoria más alta ocurrió en la región pacífica, siendo de 1,0 \% y 21,6 \% respectivamente. La región que presentó la menor tasa de mortalidad perioperatoria en la mayoría de los grupos etarios fue Bogotá, D.C., de 18 a 49 años, 0,07 \%; de 50 a 64, 0,7\%; de 65 a $80,3,0 \%$, y mayores de 80 años, 12,5\%. De igual forma, la menor tasa de mortalidad fue encontrada en la región oriental con 0,01 \% en personas entre los 10 y 18 años, rango de edad que también presenta la menor tasa de mortalidad en todas las demás regiones (tabla 3 ).

Cuando se evaluó la tasa de mortalidad perioperatoria por año (tabla 4), se observó que la mortalidad fue mayor en el año $2016(0,7 \%)$ y menor

Tabla 1. Tasa de mortalidad perioperatoria por sexo.

\begin{tabular}{lcc}
\hline \multicolumn{1}{c}{ Edad (años) } & Mujeres (\%) & Hombres (\%) \\
\hline $0-1$ & $3,43(\mathrm{n} \neq=146)$ & $2,91(\mathrm{n} \neq=206)$ \\
$1-5$ & $0,07(\mathrm{n} \neq=1371)$ & $0,33(\mathrm{n} \neq=1536)$ \\
$5-10$ & $0,08(\mathrm{n} \neq=6290)$ & $0,01(\mathrm{n} \neq=8419)$ \\
$10-18$ & $0,03(\mathrm{n} \neq=21.895)$ & $0,02(\mathrm{n} \neq=23.866)$ \\
$18-50$ & $0,09(\mathrm{n} \neq=67.479)$ & $0,10(\mathrm{n} \neq=69.050)$ \\
$50-64$ & $1,13(\mathrm{n} \neq=10.414)$ & $1,23(\mathrm{n} \neq=9166)$ \\
$65-80$ & $5,12(\mathrm{n} \neq=4194)$ & $5,99(\mathrm{n} \neq=3742)$ \\
$>80$ & $12,55(\mathrm{n} \neq=1147)$ & $17,31(\mathrm{n} \neq=959)$ \\
\hline
\end{tabular}

‡ Número de procedimientos.

Tabla 2. Tasa de mortalidad perioperatoria por grupo etario.

\begin{tabular}{lcc}
\hline Edad (años) & Porcentaje & IC $_{95 \%}$ \\
\hline $0-1$ & 3,13 & $1,74-5,56$ \\
$1-5$ & 0,21 & $0,09-0,46$ \\
$5-10$ & 0,04 & $0,02-0,09$ \\
$10-18$ & 0,02 & $0,01-0,04$ \\
$18-50$ & 0,10 & $0,08-0,12$ \\
$50-64$ & 1,12 & $0,99-1,28$ \\
$65-80$ & 5,53 & $5,05-6,06$ \\
$>80$ & 14,38 & $12,97-15,92$ \\
\hline
\end{tabular}


Tabla 3. Tasa de mortalidad perioperatoria por grupo etario y regiones.

\begin{tabular}{|c|c|c|c|c|c|c|}
\hline Edad (años) & $\begin{array}{c}\text { Región Atlántica } \\
(\%)\end{array}$ & $\begin{array}{c}\text { Bogotá DC } \\
(\%)\end{array}$ & $\begin{array}{c}\text { Región Central } \\
(\%)\end{array}$ & $\begin{array}{c}\text { Región Oriental } \\
(\%)\end{array}$ & $\begin{array}{c}\text { Región Pacífica } \\
(\%)\end{array}$ & $\begin{array}{c}\text { Otras regiones } \\
(\%)\end{array}$ \\
\hline $0-1$ & $2,04(n \ddagger=50)$ & $6,89(n \ddagger=94)$ & $\begin{array}{c}3,18 \\
(n \neq=65)\end{array}$ & $\begin{array}{c}0 \\
(n \neq=97)\end{array}$ & $\begin{array}{c}2,44 \\
(n \neq=42)\end{array}$ & $\begin{array}{l}33,33 \\
(n \neq=4)\end{array}$ \\
\hline $1-5$ & $0,46 \quad(n \ddagger=218)$ & $0,17(n \ddagger=1200)$ & $0,37 \quad(n \ddagger=550)$ & $0,15(n \ddagger=652)$ & $0(n \ddagger=244)$ & $0(n \ddagger=46)$ \\
\hline $5-10$ & $0,07(n \ddagger=1349)$ & $0,06(n \ddagger=5404)$ & $0,29(n \ddagger=3474)$ & $0,03(n \neq=3205)$ & $0(n \neq=1397)$ & $0(n \neq=274)$ \\
\hline $10-18$ & $0,04(n \ddagger=5339)$ & $0,03(n \ddagger=1495)$ & $0,02(n \ddagger=11.482)$ & $0,01(n \ddagger=9694)$ & $0,02(n \ddagger=4765)$ & $0(n \neq=720)$ \\
\hline $18-50$ & $0,15(n \ddagger=16825)$ & $0,07(n \ddagger=4718)$ & $0,08(n \ddagger=30.341)$ & $0,10(n \ddagger=26.428)$ & $0,10(n \neq=13.424)$ & $0,34(n \ddagger=2344)$ \\
\hline $50-64$ & $1,52(n \neq=1936)$ & $0,76(n \ddagger=6795)$ & $1,42(n \ddagger=4571)$ & $1,12(n \ddagger=3716)$ & $1,41(n \ddagger=2307)$ & $1,16(n \neq=262)$ \\
\hline $65-80$ & $9,13 \quad(n \ddagger=693)$ & $3,02(n \ddagger=2521)$ & $7,66(n \ddagger=2067)$ & $5,64(n \neq=1442)$ & $7,64(n \ddagger=1156)$ & $1,72(n \ddagger=59)$ \\
\hline$>80$ & $17,53(n \ddagger=228)$ & $12,50(n \ddagger=594)$ & $18,39(n \ddagger=631)$ & $15,89(n \ddagger=343)$ & $21,60(n \ddagger=366)$ & $27,27(n \ddagger=14)$ \\
\hline
\end{tabular}

‡Número de procedimientos.

en 2013 (0,43\%). Así mismo, dentro del periodo del 2012 al 2016, el año con menor volumen quirúrgico fue 2016 con 0,48 apendicectomías por 1000 afiliados, presentándose la mayor frecuencia en el año 2012, con 1,94 apendicectomías por 1000 afiliados (Tabla 5).

\section{Discusión}

La tasa de mortalidad perioperatoria global de apendicectomías, encontrada entre los individuos de 1 año a 50 años de edad en nuestro estudio, fue cercana a la de otros países de ingresos medios y bajos ${ }^{10}$, con más procedimientos realizados en hombres, similar a lo hallado en otros estudios latinoamericanos, sin embargo, con una relación más cercana a las mujeres ${ }^{6,8,11}$. En cuanto a la edad, hubo mayor número de apendicectomías en personas adultas entre los 18 y los 49 años, con tasa de mortalidad perioperatoria mayor en los extremos de la vida, lo cual concuerda con otros estudios en los que se halla una asociación fuerte de mortalidad en pacientes menores de 1 año y mayores de 65 años ${ }^{12}$.

El tipo de cirugía más realizada fue la apendicectomía abierta o por laparotomía, advirtiendo que el poco uso de la herramienta laparoscópica puede ser explicado por el contexto socioeconómico que limita a muchos de los centros de salud en países en vías de desarrollo, debido a la baja disponibilidad de los recursos físicos y de personal capacitado para su utilización. Además, en consideración con diferentes estudios donde se
Tabla 4. Tasa de mortalidad perioperatoria por año.

\begin{tabular}{ccc}
\hline Año & Porcentaje & IC $_{95 \%}$ \\
\hline 2011 & 0,54 & $0,48-0,61$ \\
2012 & 0,45 & $0,40-0,52$ \\
2013 & 0,43 & $0,37-0,49$ \\
2014 & 0,48 & $0,42-0,56$ \\
2015 & 0,49 & $0,42-0,57$ \\
2016 & 0,70 & $0,57-0,87$ \\
\hline
\end{tabular}

Tabla 5. Volumen quirúrgico de apendicectomías por 1000 afiliados.

\begin{tabular}{cc}
\hline Año & Volumen \\
\hline 2012 & 1,94 \\
2013 & 1,92 \\
2014 & 1,57 \\
2015 & 1,39 \\
2016 & 0,48 \\
\hline
\end{tabular}

expone que, con este tipo de cirugía se disminuye el número de complicaciones relacionadas y mortalidad $^{4,5}$, el disponer de este tipo de herramientas afecta directamente la posibilidad de disminuir la tasa de mortalidad perioperatoria de forma considerable, como lo evidenciado en regiones como la oriental y Bogotá, D.C., que cuentan con la posibilidad de utilizar este método quirúrgico con mayor frecuencia.

Es importante destacar que, más de un tercio de las apendicectomías analizadas se realizaron en Bogotá, D.C., siendo la ciudad que cuenta con 
aproximadamente un $15 \%$ de la población total de Colombia ${ }^{13}$ y la región atlántica presentó la mayor tasa de mortalidad perioperatoria en la mayoría de los grupos etarios, y la menor tasa en menores de 1 año, situación que se invierte en Bogotá D.C., donde se presentó la mayor mortalidad en este último grupo etario, a pesar de que cuenta con la menor tasa de mortalidad perioperatoria en la mayoría de sus grupos etarios.

En varias regiones no se encontraron datos de muertes perioperatorias registradas, lo cual puede tener diferentes explicaciones; una posibilidad es el bajo número de apendicectomías realizadas en aquellos departamentos; una segunda explicación se relaciona con el escaso volumen de afiliados de las EPS en las regiones de las cuales obtuvimos información para el presente estudio que, a su vez, se puede correlacionar con un menor número de habitantes en estas mismas regiones, así como con un reducido número de centros asistenciales de segundo nivel.

En cuanto al volumen quirúrgico, no se encontró correlación con la tasa de mortalidad perioperatoria por año, dado que no aumenta la mortalidad con el incremento concomitante del volumen quirúrgico.

Entre las fortalezas de este estudio destacamos que, se posiciona como uno de los primeros acercamientos en la literatura para evaluar la tasa de mortalidad perioperatoria en apendicectomías realizadas en Colombia, con un gran tamaño de muestra y fuentes de datos nacionales, lo cual permitiría extrapolar los resultados a la población colombiana en general.

Dentro de las limitaciones del estudio podemos encontrar que los resultados se encuentran sujetos a la correcta compilación de datos y que únicamente se analizó el régimen contributivo, dejando de lado el régimen subsidiado de aseguramiento en salud. En la población total inicial, se pueden encontrar sujetos a quienes posiblemente se les realizó una apendicectomía previa al 2011, por lo cual no se tuvieron en cuenta. Por otro lado, teniendo en consideración que los datos utilizados para la tasa de mortalidad perioperatoria fue la mortalidad en la población colombiana, proporcionada por la base de datos utilizada, varias de estas muertes pueden deberse a otro motivo y no necesariamente a la cirugía.

\section{Conclusiones}

La tasa de mortalidad global de apendicectomías es comparable con la de otros países en condiciones similares de ingresos medios y bajos. Este estudio permite una aproximación inicial para generar estrategias de vigilancia e intervención oportuna que conlleven a la reducción de la mortalidad de una de las cirugías más comúnmente practicadas en Colombia.

\section{Cumplimiento de normas éticas}

Consentimiento informado: Este trabajo se basa en la revisión de una base de datos, por lo que no se requiere consentimiento informado por parte de los pacientes. El estudio fue aprobado por el Comité de ética de la Facultad de Medicina de la Universidad Nacional de Colombia.

Conflictos de interés: Los autores declaran no presentar ningún conflicto de intereses.

Fuente de financiación: Para la realización del estudio se utilizaron recursos de la Universidad Nacional de Colombia.

Contribución de los autores: Concepción y diseño del estudio, adquisición, análisis e interpretación de datos, redacción del manuscrito y revisión crítica: Giancarlo Buitrago-Gutiérrez.

Análisis e interpretación de datos, redacción y revisión crítica: Jimmy Santiago Castellanos-Méndez, Nicolle Simmonds-Campbell.

\section{Referencias}

1. Ávila M, García M. Apendicitis aguda: revisión de la presentación histopatológica en Boyacá, Colombia. Rev Colomb de Cir. 2015;30:125-130.

2. Stewart B, Khanduri P, McCord C, Ohene M, Uranues S, Vega-Rivera F, et al. Global disease burden of conditions requiring emergency surgery. Brit J Surg. 2013;101:922. https://doi.org/10.1002/bjs.9329

3. Bejarano M, Gallego CX, Gómez JR. Frecuencia de abdomen agudo quirúrgico en pacientes que consultan al servicio de urgencias. Rev Colomb Cir. 2011;26:33-41.

4. Toro J, Barrera Ó, Morales C. Superioridad clínica de la apendicectomía laparoscópica sobre la técnica abierta: ¿adopción lenta de un nuevo estándar de tratamiento?. Rev Colomb de Cir. 2017;32:32-39.

https://doi.org/10.30944/20117582.5 
5. Souza L, Martínez J. Apendicitis aguda. Manejo quirúrgico y no quirúrgico. Rev Med Inst Mex Seguro Soc. 2016;55:76-81.

6. Bustos N, Cabrera E, Castaño-Castrillón J, Jaimes A, Pérez J, Rincón D, et al. Epidemiología de pacientes con diagnóstico de apendicitis aguda no especificada, egresados del servicio de hospitalización del Hospital Infantil Universitario "Rafael Henao Toro" de la ciudad de Manizales (Colombia) 2011- 2012: estudio de corte transversal. Archivos de Medicina (Col). 2015;15:6776. https://doi.org/10.30554/archmed.15.1.731.2015

7. González J. Revisión bibliográfica apendicitis aguda. Medicina Legal de Costa Rica. 2012;29:83-90.

8. Rodríguez-FernandezZ.Complicaciones dela apendicectomía por apendicitis aguda. Rev Cubana Cir. 2010;49(2). Disponible en: http://scielo.sld.cu/scielo.php?script=sci_arttext\&pid=S0034-74932010000200006

9. Ferris M, Quan S, Kaplan B, Molodecky N, Ball C, Chernoff $\mathrm{G}$, et al. The global incidence of appendicitis. Ann Surg. 2017;266:237-41.

https://doi.org/10.1097/SLA.0000000000002188
10. Ng-Kamstra J, Arya S, Greenberg S, Kotagal M, Meara J, Shrime M. Perioperative Mortality Rates: A systematic review and meta-analysis of the literature from low- and middle-income countries. J Am Coll Surgeons. 2017;225:107.

https://doi.org/10.1016/j.jamcollsurg.2017.07.236

11. Gavilán R. Morbilidad y mortalidad por apendicitis aguda en el hospital integral comunitario del municipio Monteagudo (2006-2008). MEDISAN. 2010;14.2010-16. Disponible en: http://scielo.sld.cu/scielo.php?script=sci_arttext\&pid=S1029-30192010000800010\&lng=es

12. Whitlock E, Feiner J, Chen L. Perioperative Mortality, 2010 to 2014: A Retrospective Cohort Study Using the National Anesthesia Clinical Outcomes Registry. Anesthesiology. 2015;123:312-21.

https://doi.org/10.1097/ALN.0000000000000882

13. DANE. Censo Nacional de Población y Vivienda [base de datos en línea]. Bogotá: DANE; 2018. Fecha de consulta: 19 de marzo de 2020. Disponible en: https:// www.dane.gov.co/index.php/estadisticas-por-tema/ demografia-y-poblacion/censo-nacional-de-poblacion-y-vivenda-2018/cuantos-somos 